\title{
COVID-19 reveals influence of physical activity on symptom severity in hereditary spastic paraplegia
}

\author{
Lotte van de Venis ${ }^{1} \cdot$ Bart P. C. van de Warrenburg ${ }^{2} \cdot$ Vivian Weerdesteyn $^{1} \cdot$ Bas J. H. van Lith ${ }^{1}$. \\ Alexander C. H. Geurts ${ }^{1,3}$. Jorik Nonnekes ${ }^{1,3}$
}

Received: 13 May 2020 / Revised: 18 June 2020 / Accepted: 18 June 2020 / Published online: 7 July 2020

○) Springer-Verlag GmbH Germany, part of Springer Nature 2020

\section{Dear Sirs,}

Hereditary spastic paraplegia (HSP) is a neurodegenerative disorder, characterized by progressive spasticity and muscle weakness of both lower extremities [1]. Spasticity-related symptoms such as muscle stiffness and gait impairments are common and disabling in HSP [2]. Moreover, patients experience a substantial burden from both physical and mental fatigue [2]. Our clinical experience is that physical activity positively impacts on these symptoms, whereas psychological stress may impact negatively. This has, however, not been formally investigated. The (partial) lockdown due to the COVID-19 pandemic has profoundly changed people's normal routine, assumably reducing levels of physical activity and increasing psychological stress [3], thereby creating an opportunity to explore the influence of these changes on symptom severity in HSP.

We conducted a web-based survey among people with pure HSP [2] in the Netherlands. An invitation was sent to participants from our previous survey $(n=109)$, which was approved by our regional medical-ethics committee. Participants were asked to rate possible changes in levels of

Alexander C. H. Geurts and Jorik Nonnekes contributed equally to this work

Lotte van de Venis

lotte.vandevenis@radboudumc.nl

1 Donders Institute for Brain, Cognition and Behavior, Department of Rehabilitation, Center of Expertise for Parkinson and Movement Disorders, Radboud University Medical Center, Nijmegen, The Netherlands

2 Donders Institute for Brain, Cognition and Behavior, Department of Neurology, Center of Expertise for Parkinson and Movement Disorders, Radboud University Medical Center, Nijmegen, The Netherlands

3 Department of Rehabilitation, Sint Maartenskliniek, Ubbergen, The Netherlands physical activity, psychological stress, and symptom severity on a 5-point Likert scale. They were invited and completed the questionnaire during the fifth week of the partial lockdown in the Netherlands. Descriptive statistics were used to analyze the primary data. Additionally, chi-square tests (or Fisher-exact-tests if appropriate) were used to test whether changes in physical activity and psychological stress were associated with changes in symptom severity $(p<0.05)$. When both physical activity and psychological stress were associated with a specific change in symptom severity, multivariate logistic forward regression analysis was applied to correct for collinearity of these independent determinants.

Fifty-eight participants returned a completed survey. Their average age was 57 years (range $30-77$ ) and $47 \%$ was male. A reduction of physical activities was reported by $74 \%$ (33\% strong decrease, $41 \%$ mild decrease), whereas $19 \%$ reported no change and $7 \%$ mild increase. An increase in psychological stress was reported by $43 \%$ (3\% strong increase, $40 \%$ mild increase), $50 \%$ reported no change, and $7 \%$ decrease (2\% strong, $5 \%$ mild). The majority reported a general increase in symptom severity (Fig. 1).

Participants with reduced physical activity more often experienced increased muscle stiffness $(p=0.001)$, pain $(\mathrm{p}=0.004)$, physical fatigue $\left[\chi^{2}(1)=4.680, p=0.031\right]$, and gait impairments $\left[\chi^{2}(1)=5.129, p=0.024\right]$ compared to those with no change or an increase in physical activity (Fig. 2). The same trend was seen for balance impairments $\left[\chi^{2}(1)=3.291, p=0.070\right]$. Those who reported increased levels of psychological stress more often reported an increase in muscle stiffness $\left[\chi^{2}(1)=4.612, p=0.032\right]$, pain $\left[\chi^{2}(1)=3.943, p=0.047\right]$, and mental fatigue $\left[\chi^{2}(1)=6.234\right.$, $p=0.013]$. Forward regression analysis of muscle stiffness and pain revealed that only decreased physical activity was independently associated with an increase in muscle stiffness $\left[R^{2}=0.236(p<0.001)\right]$ and pain $\left[R^{2}=0.193(p=0.003)\right]$.

Six participants were treated with intramuscular botulinum toxin injections to reduce spasticity-related 


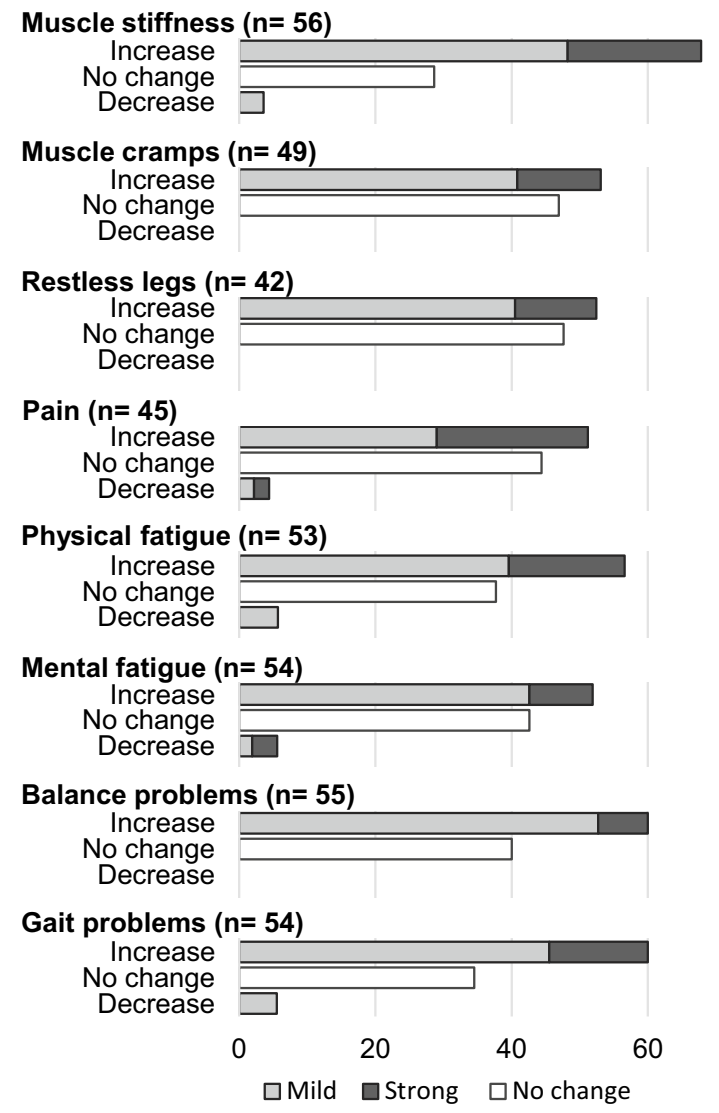

Fig. 1 The impact of the COVID-19 partial lockdown measures on spasticity-related symptoms in people with pure HSP. Participants reported whether the experienced symptom severity increased (mild or strong), decreased (mild or strong) or did not change. Only those participants who experienced a specific symptom (either before or after the lockdown) are included in the figure

symptoms. During the lockdown, treatment continued in five participants.

The partial lockdown in the Netherlands due to the COVID-19 pandemic resulted in a reduction of physical activity in the majority of participants with HSP, which proved to be associated with increased muscle stiffness, pain, physical fatigue and gait impairments. This result is coherent with findings in other chronic (neurodegenerative) conditions [4] and underscores the potential impact of physical activity on symptom severity in people with HSP. Future studies may investigate whether the present findings can be extended to other conditions resulting in spastic paraparesis (e.g. multiple sclerosis and primary lateral sclerosis). Future studies may also evaluate the effect of interventions targeting daily physical activity in this population, preferably including objective outcomes, which were lacking in the present study. Another limitation is the lack of comparison between current and previous clinical status, which was not possible due to the
Impact of physical activity on symptom severity
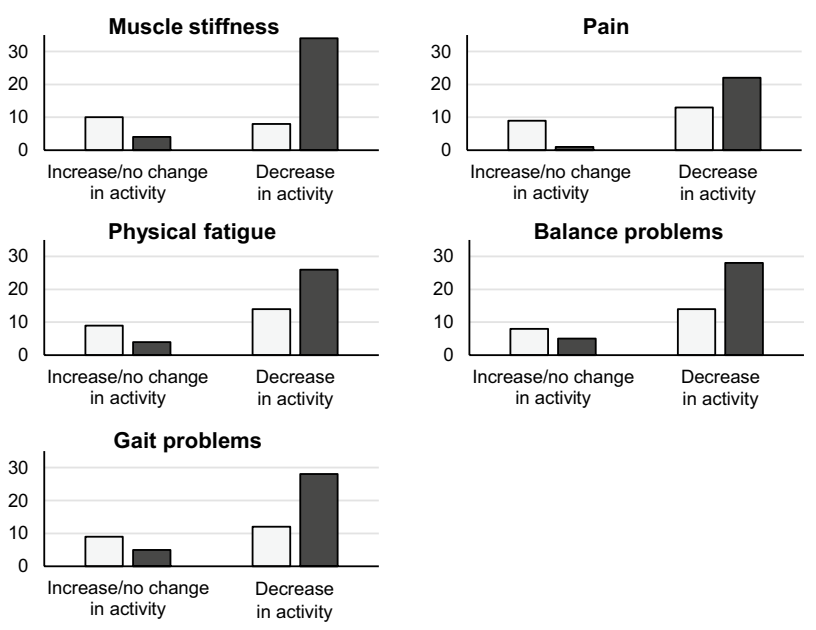

$\square$ Decrease/no change in symptom severity

$\square$ Increase in symptom severity

Impact of psychological stress on symptom severity

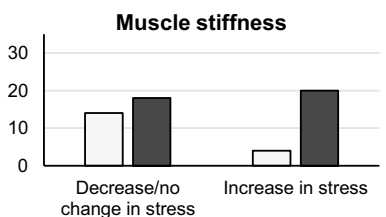

Pain
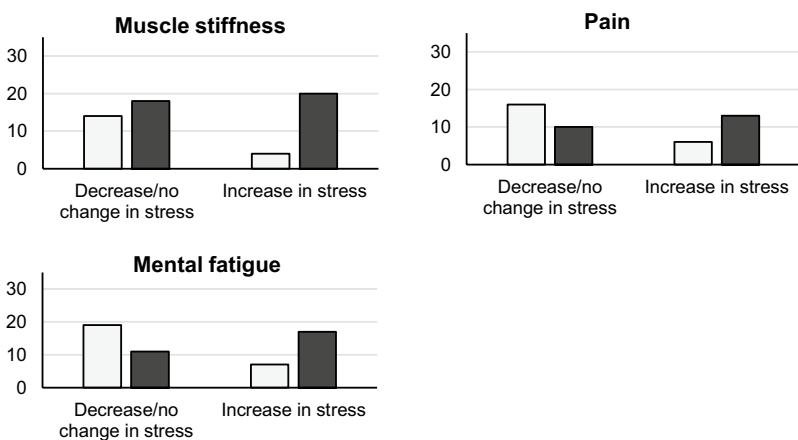

$\square$ Decrease/no change in symptom severity

$\square$ Increase in symptom severity

Fig. 2 The impact of physical activity and psychological stress on symptom severity in people with pure HSP. $Y$-axis represents number of people with HSP who experienced the symptom

lockdown restrictions. An additional limitation is the risk of selection bias, which may have resulted in an overestimation of changes in physical activity, psychological stress, and/or symptom severity. The question remains whether people with HSP are able to return to 'baseline' levels of functioning after release of the lockdown and expected increase in physical activity.

Acknowledgements Several authors (BW, AG, JN) of this publication are members of the European Reference Network for Rare Neurological Diseases- Project ID No 739510.

Author contributions $\mathrm{LV}$, JN: collecting survey data, interpreting survey data, and drafting the manuscript. BL, BW, VW, AG: critical revision of manuscript.

Funding This study was supported by the Jacques and Gloria Gossweiler Foundation. 


\section{Compliance with ethical standards}

Conflicts of interest The authors declare that they have no conflict of interest.

Ethical standard statement This study was in accordance with local ethical guidelines.

\section{References}

1. Shribman S et al (2019) Hereditary spastic paraplegia: from diagnosis to emerging therapeutic approaches. Lancet Neurol $18: 1136-1146$
2. van Lith BJH et al (2020) Experienced complaints, activity limitations and loss of motor capacities in patients with pure hereditary spastic paraplegia: a web-based survey in the Netherlands. Orphanet J Rare Dis 15(1):64

3. Helmich RC, Bloem BR (2020) The impact of the COVID-19 pandemic on Parkinson's disease: hidden sorrows and emerging opportunities. J Parkinsons Dis 10(2):351-354

4. van der Kolk NM et al (2019) Effectiveness of home-based and remotely supervised aerobic exercise in Parkinson's disease: a double-blind, randomised controlled trial. Lancet Neurol 18(11):998-1008 